\title{
La vulnerabilidad urbana: entre reducción de riesgo y emancipación social. Ejemplos en Venezuela
}

\author{
Julien Rebotier \\ CNRS, Pau, Francia. \\ Email: julien.rebotier@cnrs.fr
}

\begin{abstract}
Resumen: ${ }^{1}$ La noción de vulnerabilidad emerge de corrientes académicas críticas, a partir de finales de los años 1960. Como concepto progresista del análisis de riesgo, arraiga los factores explicativos de las situaciones de riesgo en la organización de la sociedad y las relaciones sociales. Favorece el análisis de la estructura causal de la construcción de riesgos, alejándose de la amenaza natural, para entender las lógicas sociales imperantes. En contextos urbanos sumamente discriminatorios e históricamente desiguales, tal perspectiva resulta emancipadora y se basa tanto en aspectos materiales como inmateriales. Cuestionar la construcción de riesgo es una manera de cuestionar los fundamentos discursivos y materiales de un orden socio-espacial urbano injusto. De ahí que no se busca eludir los impactos de los eventos naturales, sino que se intenta reducir la vulnerabilidad de forma sostenible y compartida al transformar las condiciones socio-espaciales que intervienen en la construcción de riesgo.
\end{abstract}

Palabras clave: Riesgo; vulnerabilidad; dominación; políticas; Caracas.

\section{Urban vulnerability: between risk reduction and social emancipation. Examples from Venezuela.}

\begin{abstract}
Vulnerability stems from a late 1960s critical academic background. As a progressive risk analysis concept, it consists in embodying triggering factors of risk situations in social organization and social relations. It promotes the analysis of risk construction through root social and political causes, instead of focusing on hazard. In highly discriminating and historically unequal urban contexts, such a viewpoint aims to achieving poor and marginalized people emancipation on the basis of both material and immaterial levers. By dealing with social construction of risk, vulnerability addresses the material and speculative socio-spatial basis of unfair urban settlements. The main issue is not to elude hazard impacts. Rather, the point consists in reducing vulnerability in a sustainable and shared way by transforming the socio-spatial conditions that frame the construction of risks.
\end{abstract}

Key words: Risk; vulnerability; domination; politics; Caracas. 


\section{Vulnerabilidade urbana: entre a redução do risco e da emancipação social. Exemplos na Venezuela}

Resumo: Noção de vulnerabilidade emerge revisões acadêmicas atuais, a partir de final de 1960 como conceito progressivo de análise de risco, fatores explicativos raiz de situações de risco na organização da sociedade e as relações sociais. Análise favorece a estrutura causal do risco de construção, longe do perigo natural, para compreender as lógicas sociais vigentes. Em contextos urbanos altamente discriminatórias e historicamente desiguais, tal perspectiva é libertadora e baseia-se tanto em aspectos materiais e imateriais. Questionar a construção do risco é uma forma de desafiar as bases discursivas e materiais de ordem sócio-espacial urbana injusto. Por isso, não procurando evitar os impactos de eventos naturais, mas tente reduzir a vulnerabilidade dos sustentável e compartilhado por transformar as condições sócio-espaciais envolvidos na construção de risco.

Palavras-chave: Risco; vulnerabilidade; dominação; políticas; Caracas.

\section{Introducción: riesgo, ciencia y sociedad en una encrucijada}

El estudio del riesgo cuenta con más conocimientos conforme va pasando el tiempo. Por lo tanto, no van disminuyendo de manera significativa los impactos y las consecuencias de los desastres en las poblaciones y los territorios que ocupan. Mayores conocimientos sobre el riesgo no coinciden con mejores acciones en el terreno. Tal paradoja lleva a formular dos hipótesis relacionadas con la manera como se realizan los estudios de riesgo, y con el papel que desempeña la investigación sobre riesgo en los territorios estudiados.

Por un lado, el análisis clásico de los riesgos aísla los elementos relativos a la amenaza de los que se vinculan con la vulnerabilidad, dejando el trabajo de integración de los dos grupos de elementos en una incertidumbre metodológica y epistémica. Al reorganizar la concepción del riesgo a partir de la organización, del funcionamiento de la sociedad, y de su relacionar con el espacio y el medio ambiente, se ofrece la posibilidad de fundamentar el riesgo en una serie de condiciones sociales basándose en la noción de vulnerabilidad. Los factores notorios que hace falta tomar en consideración en los estudios de riesgo no sólo son materiales y visibles (como en el caso de las condiciones de exposición, o de susceptibilidad de un edificio frente a una amenaza) sino que también son inmateriales y simbólicos (como lo pueden ser relaciones sociales discriminatorias en base a criterios socio-económicos, de género o étnico, o también estatutos y posiciones de dominación o de fuerte legitimidad social). Se propone una conceptualización del riesgo mediante la vulnerabilidad para dar espacio a mecanismos explicativos, inmateriales y simbólicos, relacionados con el orden socio-espacial.

Por otro lado, es de notar que los estudios de riesgo producen un discurso sobre los riesgos. La ciencia, como actividad social, no es ajena al 
orden socio-espacial que contribuye a formatear las situaciones de riesgo. Si bien participan los factores simbólicos a las situaciones de riesgo, el discurso científico, lejos de una vana objetividad o neutralidad axiológica, también formatea el mundo interpretado. Tanto en la elaboración del objeto de estudio como en sus objetivos y en las implicaciones de sus análisis, los estudios de riesgo bien pueden consolidar o cuestionar las situaciones descritas. La ciencia se hace en sociedad, desde la formulación de la pregunta, hasta el uso que se hace del análisis. Se propone integrar la dimensión performativa de la ciencia agua arriba de los estudios de riesgo para fomentar una ciencia comprometida, es decir que asume su dimensión situada en un contexto social, y no se reduce a una función técnica y operacional.

El presente artículo tiene como objetivo demostrar la importancia de dos elementos claves en el abordaje progresista ${ }^{2}$ de la vulnerabilidad: 1 - su carácter eminentemente político, o sea cuestionado y plural (Forsyth 2012); y 2- sus fundamentos socialmente construidos.

La demostración adopta diferentes perspectivas en base a varios trabajos de campo realizados en Caracas y en Mérida (Venezuela), y se organiza en tres partes. Tras una discusión conceptual que permite darle respuesta a una de las hipótesis, la primera parte evidencia el peso de las ideas dominantes en las formas socio-espaciales de la ciudad de Caracas y en la manera como la sociedad urbana se relaciona con el medio ambiente. La segunda hace hincapié en la jerarquización de los factores de riesgo, en particular entre los mecanismos biofísicos y los demás factores, políticos y sociales, que caracterizan la vulnerabilidad. La última parte subraya la importancia de formalizar los objetivos de la gestión de riesgo, siendo la reducción de la vulnerabilidad y la garantía del funcionamiento de un sistema urbano dos objetivos diferentes, que hasta se pueden desvincular.

\section{El difícil reconocimiento de los fundamentos sociales de los riesgos}

La noción de vulnerabilidad emerge de una corriente académica crítica, a partir de finales de los años 1960. Tras la apertura de los estudios de riesgo a las dimensiones social y humana (White 1958; Burton y Kates 1964), un enfoque de economía política y de articulación escalar del análisis lleva un sector de la academia a elaborar y consolidar una noción que permite entender y explicar el riesgo a partir de las relaciones sociales (Hewitt y Burton 1971; Wisner 1976). No solamente se trata de explicar las opciones privilegiadas por los grupos o los individuos frente a una amenaza potencial. A través de la organización, la dinámica social y las relaciones situadas al medio ambiente, también se busca investigar una cadena de causas, se intenta explicar lo que hace la gente vulnerable. Más que la característica de un estado, la vulnerabilidad es un conjunto de condiciones construidas en base a las cuales grupos, individuos, sistemas urbanos... resultan 
diferentemente propensos a sufrir daños. El objetivo del enfoque crítico a los riesgos consiste en investigar las condiciones múltiples que contribuyen a la construcción de situaciones de riesgo.

El abordaje crítico de los riesgos a través de la vulnerabilidad permite articular las dinámicas socio-espaciales con las modalidades del doblamiento, es decir la manera como una sociedad se distribuye en, y se relaciona con el medio ambiente. Un modelo conceptual llamado PAR -Pressure and Release - consiste en asesar como se combinan por un lado dinámicas biofísicas y por otro lado características sociales que constan de causas profundas, de dinámicas sociales particulares al caso de estudio y de condiciones coyunturales (Wisner et al 2004 [1994]). Sigue obvia la separación moderna entre el medio físico con sus elementos de presión, de amenaza pressure, y las configuraciones sociales con sus elementos de más o menos alivio - release, pero el modelo hace hincapié en los condicionantes sociales tales como la organización, la dinámica social o la relación al espacio, más que en las dinámicas biofísicas como los estudios de riesgo -Hazard research-suelen hacerlo. El modelo PAR busca situar una sociedad en riesgo entre las dos series de elementos biofísicos y sociales, dentro de una secuencia temporal, espacial y social.

Hoy día, los debates tanto académico como social sobre el cambio climático, sobre la cuestión ambiental, y de forma más amplia, sobre los límites de la modernidad (Giddens 1994 [1991]; Beck 2003 [1986]) nutren un aggiornamento de la manera como se consideran las relaciones entre sociedades y medio ambiente (Beck 2007). Existen postulados conceptuales como la idea de naturaleza producida (Smith y O'Keefe 1980; Ekers y Loftus 2013), como las referencias a un nuevo "contrato social” (Serres 1990), o en una perspectiva más funcional y pragmática, como lo que implica el uso de las nociones de sistemas socio-ecológicos (Young et al 2006). Sin adentrarse mucho en las ramas múltiples de las diferentes maneras de conceptualizar las relaciones entre sociedad y medio ambiente, es de notar cuan importante es la voluntad de distanciarse de nociones modernas, "puras”, de diferenciación y de exclusividad, para considerar interacciones entre sociedades y medio ambiente, formas más híbridas, más complejas y de relaciones mutuas (Latour 1994). Articular las dinámicas sociales y ambientales es un desafío conceptual imperioso (Janssen y Ostrom, 2006) que fundamenta los estudios críticos de riesgo. Esa idea de co-evolución socio-ambiental contribuye a modificar la noción de vulnerabilidad y su conceptualización moderna, que diferencia sociedad y ambiente -incluyendo la del modelo PARtanto como favorece la emergencia de otros enfoques y conceptos a lo largo de las últimas décadas. Desarrollo sostenible, adaptación y resiliencia, servicios ecosistémicos y ambientales o multifuncionalidad en agricultura, para citar algunas nociones y ejemplos de sus corolarios, son relevantes tanto en el registro operacional como en la academia (aunque de forma diferenciada). Cada una de aquellas nociones trae implícitos, universos epistémicos y referencias diferentes. A menudo, se puede articular una misma noción a diferentes conjuntos de referencias. 
Ninguna de aquellas nociones tiene valor absoluto, a pesar de las veleidades de algunos sectores operacionales pero también académicos en llevar la voz cantante e imponer una interpretación puntual (Rebotier et al 2013, para la noción de resiliencia; Simonet 2009, para la noción de adaptación; Bonnal et al 2012, para la noción de servicios ambientales). La vulnerabilidad no es una excepción, y su genealogía crítica no impide una normalización e institucionalización de los años 1960 en adelante (Revet 2011). En todo caso, son varios los universos conceptuales a los cuales se puede referir la vulnerabilidad, y es fundamental situar la noción en términos epistémicos, saber quienes la usan, y en que contexto (O’Brien et al 2007).

En este contexto, un abordaje social y político de vulnerabilidad sólo considera las situaciones de riesgo, su dimensión simbólica tanto como sus implicaciones, en relación con las lógicas sociales, materiales e inmateriales, a las cuáles dichas situaciones están vinculadas. No parte de la amenaza. Dimensión importante del modelo PAR, los elementos biofísicos bien pueden constituir una serie de factores de vulnerabilidad, pero entre otros muchos. Las situaciones de riesgo (y más allá, las lógicas y problemáticas ambientales) son consideradas a partir de la organización y las relaciones sociales (Ribot 2009). De ser así, abogamos para un enfoque de geografía social y política de los riesgos que permita: 1- asesar la co-evolución socio-ambiental del poblamiento urbano, o sea la manera como sociedades y medio ambiente se transforman mutuamente, 2- afirmar el papel crítico de las ciencias sociales al asumir una autonomía conceptual en el estudio del riesgo a partir de la sociedad y no en términos de amenaza ni de dinámicas biofísicas, 3defender una perspectiva no solamente descriptiva sino emancipadora y transformadora de los estudios de riesgo al buscar dentro del orden socio-espacial “¿que hace la gente vulnerable?”, como una legado de los académicos "riesgólogos" ( $\mathrm{La} \mathrm{Red}^{3}$ ) y de los de la reducción de desastres (Gaillard y Mercer 2013).

La presente reflexión sigue los pasos de Maurice Godelier (1984) cuando caracteriza las dinámicas ambientales y sociales como mutuamente constitutivas. Pensar el riesgo más allá de la separación moderna entre sociedad y medio ambiente lleva a conceptualizarlo como construcción social, tanto material como inmaterial (García Acosta 2005), en la cual los elementos biofísicos entran en consideración conjuntamente con demás factores que hace falta caracterizar y jerarquizar. En referencia a la producción del espacio (Lefebvre 2000 [1974]) el estudio crítico de la vulnerabilidad se puede articular a un modelo de producción de riesgo (Rebotier 2012a). De esta forma, el objeto crítico de la investigación es analítico, consiste en la identificación y la priorización de los factores de vulnerabilidad en los contextos estudiados (Rebotier 2012b), y es performativo, en vista a una reducción sostenible de la vulnerabilidad mediante una transformación de la relaciones sociales y de las formas del poblamiento en las cuales se originan las condiciones de vulnerabilidad. 


\section{Entre ideal y material, la pluralidad de los factores de riesgo}

Se toma como base un trabajo de geografía histórica que consiste en afianzar un análisis sobre las representaciones dominantes de Caracas con un estudio de la morfología urbana de la ciudad a partir de la época colonial (Rebotier 2011a). Es obvio el grado de interconexión entre, por un lado, una ideología urbana caracterizada por las dimensiones inmateriales de las relaciones sociales, al espacio y al medio ambiente en Caracas, y por otro lado la distribución y las formas de la urbanización de la capital venezolana y su evolución. Al considerar aquellas dos variables, se puede dividir en tres grandes fases el período que se extiende de 1567 y la creación de Caracas al comienzo del siglo 21.

Hasta los años de las guerras de independencia, entre 1811 y 1820, la ciudad se caracteriza por dinámicas demográfica y espacial relativamente bajas. Doscientos años después de su creación, no pasa de 25000 habitantes y a duras penas desborda el sitio original, circundado por colinas y quebradas al pie del Ávila (Figura 1). En 1777, Venezuela se vuelve capitanía general, y en menos de tres décadas, la ciudad pasa los 40000 habitantes. Se densifica más de lo que se extiende: pasa la superficie urbanizada de 327ha en 1770 a 347ha en 1800. El orden socio-espacial urbano reproduce la estructuración de una sociedad jerarquizada, en función de la distancia a la plaza Mayor y al corazón del poder. Se notan técnicas de transformación del medio ambiente poco avanzadas, y una concepción de la naturaleza entre superstición y pragmatismo que impide que las implicaciones ambientales de la urbanización sean significativas a lo largo de aquellos dos siglos y medio. Por ejemplo, después del sismo de San Bernabé, en 1641, el obispo Fray Mauro de Tovar se enfrenta al gobernador Ruy de Fuenmayor que, junto con el cabildo de Caracas, quiere desplazar la ciudad hacia el Este, en terrenos supuestamente menos expuestos. El clero hace impedir la mudanza, por orden de la corona, y mantiene el casco histórico de la ciudad en el mismo lugar. Sin bien por superstición se asimila el sismo a la ira divina, el pragmatismo también justifica mantener la ciudad en su lugar, conjuntamente con las posiciones de poder de la Iglesia en la capital, son sus respetivos terrenos y edificios. A pesar de la densificación de la ciudad al final del período, las interacciones entre sociedad y medio ambiente son de baja intensidad, y se caracterizan por una capacidad de intervención débil, un orden socio-espacial desigual del esquema urbano, y una representaciones de la naturaleza dominada por la superstición, pero no desprovistas de pragmatismo (Musset 2002). 
Figura 1: “Mapa Exacto” de Caracas (1772)

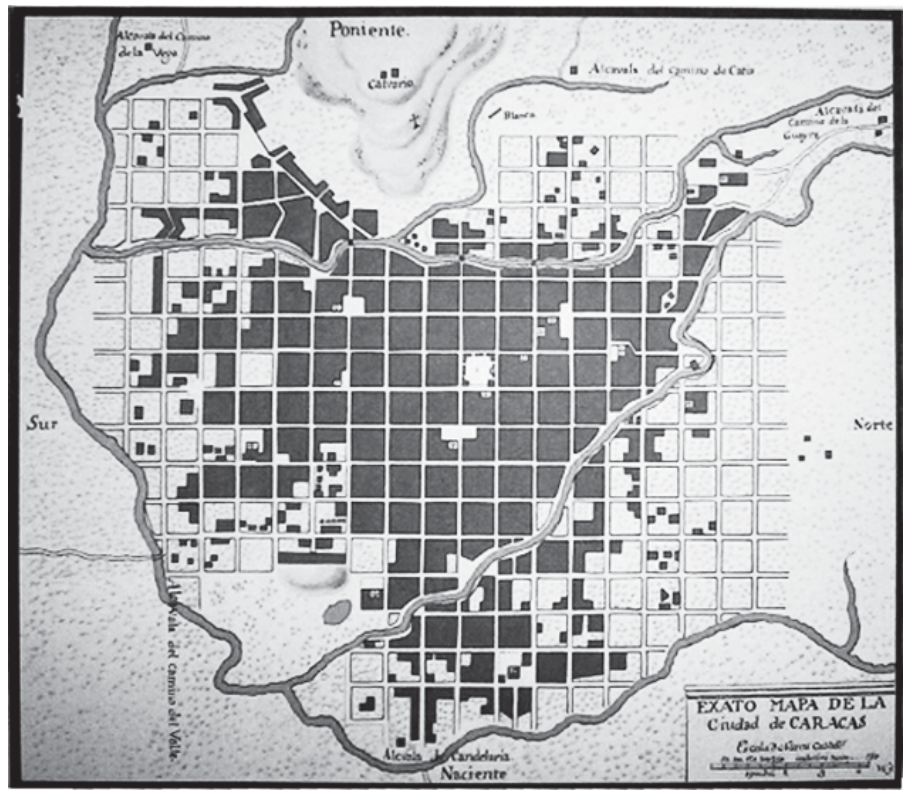

(Fuente: de Armas Chitty 1967)

Las guerras de independencia acaban con el débil crecimiento urbano. Solo se alcanza la población de 1800 en los años 1870, pero en términos morfológicos, en el último cuarto del siglo 19, el gobierno modernizador de Guzmán Blanco complementa las expansiones este y oeste de Caracas en el valle. En la primera década del siglo 20, se construye la primera urbanización en la ribera derecha del río Guaire que limitaba hasta entonces la expansión sur del centro histórico (Figura 2). A finales de los años 1900, Caracas ocupa 500ha y cuenta 90000 habitantes. Dos décadas después, alcanza los 900ha, pero a penas pasa de 100000 habitantes. En una lógica de modernización y de extensión, marcada por el alejamiento constante de las urbanizaciones, crece más la ciudad en el espacio de lo que aumenta su demografía. Empieza una redistribución de la población en unidades socialmente homogéneas, en loteos y urbanizaciones que serán el motor de la extensión urbana de Caracas en el tercer período que se describe a continuación (Fogelson 2005). Las capacidades técnicas de intervención del medio ambiente se hacen algo más notorias, pero más que todo, las representaciones de las relaciones al ambiente y la fe en la capacidad de intervención de la sociedad sobre la naturaleza cambian en relación con el período anterior. La segunda mitad del siglo 19 es el período de la modernización y del positivismo en Caracas. De la superstición, las ideas dominantes pasan a la dominación de la naturaleza, a la contención de las amenazas, y luego a su erradicación. Bolívar, 
Páez y Guzmán Blanco permiten ilustrar aquellas concepciones. En las ruinas de la Caracas de 1812, después de un sismo potente y destructor, Bolívar pronuncia la frase, hoy día famosa: "Si la naturaleza se opone, lucharemos contra ella, y haremos que nos obedezca”. En 1832, frente a la amenaza de una epidemia de cólera, el presidente Páez decreta la cuarentena de los navíos provenientes de Europa ${ }^{4}$, y el mismo año se inician visitas sanitarias para circunscribir y controlar el virus. La modernización de los espíritus atraviesa el siglo y culmina con el Ilustre Americano. En 1880, Antonio Guzmán Blanco adopta un código de salubridad ${ }^{5}$ con el fin de erradicar amenazas sanitarias objetivadas en nuevas categorías, medibles y controlables. En esa época, "se considera la naturaleza como una fuente de oportunidades que se podría transformar en tierra civilizada, en el reino del cálculo y de la abstracción, en la cúspide del cuál se allá la metrópolis” (González Casas 2002: 221). Si bien evolucionan lentamente las técnicas de intervención y la capacidad de transformar el medio ambiente (la morfología urbana evoluciona relativamente poco, y solo al final del período), es obvio el cambio de concepciones y representaciones de la naturaleza y de las relaciones al ambiente. Se consolidan las ideas de control de la naturaleza y de erradicación de las amenazas, siendo el higienismo una ilustración de aquellas ideas. La evolución de la ideología urbana es anterior a las transformaciones significativas de la morfología urbana. Con la era del petróleo, se revierte esta relación en el último período analizado, hasta llegar a la ciudad de "Caracas la horrible” (Buitrago Segura 1980).

\section{Figura 2: Primeras urbanizaciones en la ribera derecha del río Guaire, al Sur (1906)}

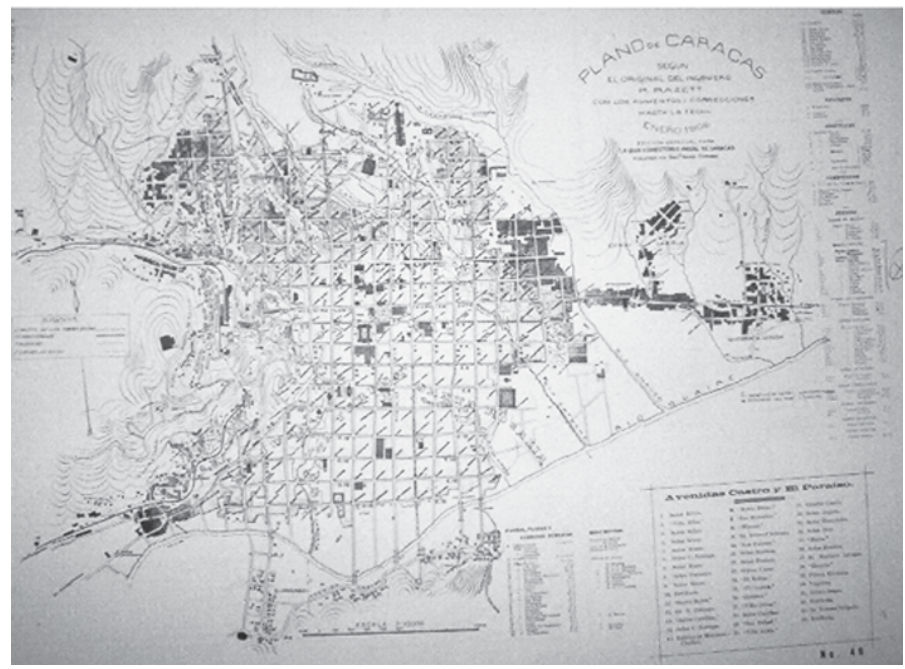

(Fuente: de Armas Chitty 1967) 
La economía venezolana se convierte al petróleo en la década 1920, iniciándose la época del petro-estado. Al apoyarse en los recursos petroleros, y con los avances tecnológicos que dan pasos de gigantes, Caracas conoce una explosión tanto demográfica como espacial a partir de mediados del siglo 20. El estado potente interviene mucho en la urbanización del valle de Caracas (no hay nada imposible para un "estado mágico”, Coronil 1997), tanto durante la dictadura como en tiempos ulteriores de democracia. La dictadura de Marcos Pérez Jiménez en los años 1950 se conoce como "los años del buldózer" durante los cuáles se busca erradicar ranchos y viviendas precarias construidas en las colinas en respuesta a la carencia de viviendas formales. En 1950, la ciudad cuenta medio millón de habitantes, y una década después, pasa del millón de habitantes. La explosión demográfica es también la de la extensión urbana. Se embaúlan los riachuelos en las laderas del Ávila, se rellenan las quebradas, y se arrasan las cumbres de las colinas para la construcción (Castillo d’Imperio 1990). El régimen democrático iniciado en 1958 enfrenta el boom demográfico incentivando la urbanización tanto del valle (no quedan espacios significativos libres en el fondo del valle a mediados de los años 1970) como de las colinas. Para muchos también, es el período de la construcción y ocupación de los ranchos en los cerros (Figura 3). A mediados de los años 1980, el volumen del material desplazado para urbanizar el valle de Caracas representa más de 20 veces el volumen afectado por todos los deslizamientos y flujos torrenciales del período pre-hispánico (Singer 1983).

\section{Figura 3: El valle de Caracas, desde el Oeste, Barrio Mario Briceño}

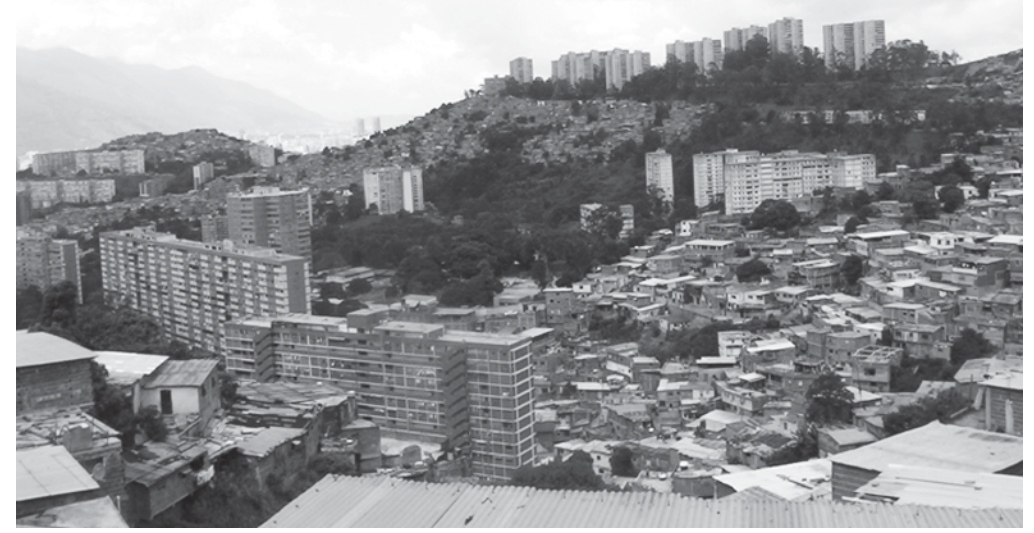

(Fuente: foto del autor, 2005)

Los saltos demográfico y morfológico resultan impresionantes, y de la ilusión de potencia, de la ciudad como escaparate de lo moderno y civilizado, se está frente a los límites obvios de la concentración y de la urbani- 
zación en un valle atravesado por fallas geológicas, y circundado por colinas cubiertas de material poco consolidado, propenso a los deslizamientos. En la creencia en la técnica y en la certidumbre en la dominación de la naturaleza, se abren brechas a partir de los años 1970. Son contemporáneas de una consciencia ambiental que se va consolidando a escala global, y que nortea las movilizaciones locales urbanas en Venezuela. Sin embargo, las iniciativas son fragmentadas, poco decisivas, y deben contar con una fuerte inercia tanto de las formas urbanas de poblamiento como de las representaciones dominantes de las relaciones entre sociedad y medio ambiente. En 1972, se crea un cinturón verde alrededor de la aglomeración de Caracas, y se inaugura un ministerio del ambiente en 1976. En una novela bandera de la literatura venezolana, Rómulo Gallegos restituye el momento de transición entre un país rural tradicional y la tendencia moderna y urbana que se dibujaba en los años 1920 (Gallegos 1929). Doña Bárbara es alegórica de los grandes virajes que vive el país en la aurora del período petrolero, tanto en la morfología de su capital, como en las ideas y representaciones que se tiene de la sociedad y de las relaciones al campo, a la naturaleza o al medio ambiente.

No existen acciones que no tengan correspondencias en las ideas (Godelier 1984). No existen modalidades de poblamiento urbano involucrando situaciones de riesgo que no tengan parte de su explicación en la manera como se conciben las relaciones entre sociedades, espacio y medio ambiente. De igual modo, las formas, la distribución y las implicaciones discriminadas de los riesgos tienen correspondencias tanto con la organización y el funcionamiento de la sociedad, como con las ideas dominantes de las relaciones sociales al ambiente. Estudiar los procesos de producción de la vulnerabilidad urbana social y espacialmente diferenciada obliga a considerar un orden socio-espacial discriminatorio, tanto material como inmaterial.

\section{Jerarquizar factores obvios y otros no tan evidentes en el estudio de riesgo}

Al estudiar casos de deslizamientos en Caracas, es posible deconstruir algunos tópicos sobre los riesgos ambientales, como: la vulnerabilidad se puede reducir aproximadamente a la pobreza, o al contener los movimientos de tierra, se controlan los riesgos. No se trata de analizar la distribución diferenciada de riquezas basada en una correspondencia entre vulnerabilidad y pobreza, sino que se propone priorizar elementos políticos, económicos e institucionales en el proceso que lleva a la construcción de las situaciones de riesgo estudiadas, en margen de la mecánica geotécnica de los deslizamientos.

Dentro del Distrito Metropolitano de Caracas, la distribución de los deslizamientos existentes o potenciales (manchas oscuras en la Figura 4) no se corresponde necesariamente con la distribución de los sectores pre- 
carios de barrios de ranchos (manchas más claras, sobrepuestas). Por ser acomodados los sectores de la California Sur, de Santa Mónica, o más aún, de Los Naranjos (Figura 4) presentan casos notorios de deslizamiento.

\section{Figura 4: Deslizamientos, barrios de ranchos y sectores acomodados en Caracas}

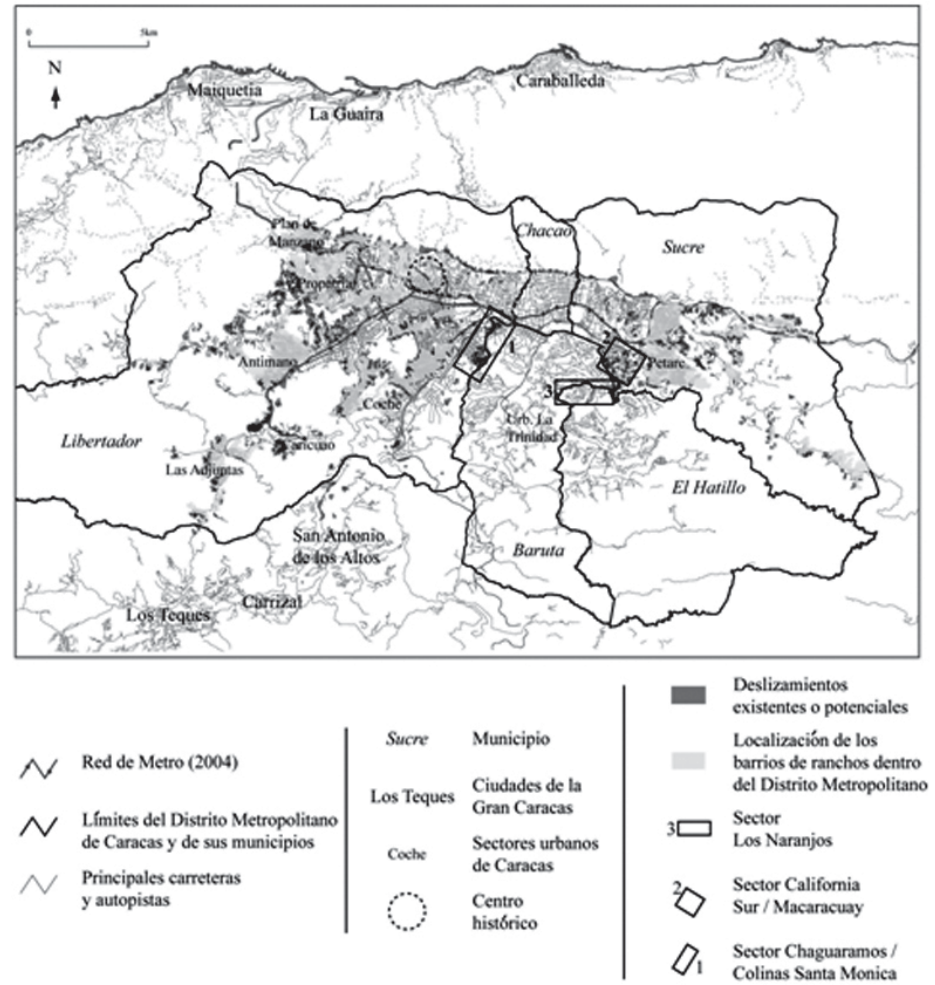

(Fuente: adaptado de Rebotier 2008)

En el caso de la urbanización Santa Mónica, es posible evidenciar factores políticos que han permitido un desarrollo urbano en un sector originalmente no autorizado para la construcción, debido a las condiciones del terreno: pendiente fuerte y material meteorizado, no consolidado. Gracias a ordenanzas urbanas especiales, se autorizan las obras en este sector en los años 1970, a pesar de la experticia contraria de geotécnicos ${ }^{6}$. Desde los años 1960, las ordenanzas especiales son el proceso normal de reglamentación de la urbanización de Caracas, pese a algunas iniciativas de 
coordinación, como por ejemplo la Oficina Metropolitana de la Planificación Urbana que desaparece en los años 1990. Mediante arbitrajes, negociaciones y acuerdos, se liberan espacios estructuralmente peligrosos para la urbanización en un contexto en el cuál "cada metro cuadrado se puede vender”. Santa Mónica no es una invasión pobre. Es un sector de clase media cuya existencia se debe a ordenanzas especiales, tomado a las vertientes de las colinas de Bello Monte, y hoy día particularmente expuesto a deslizamientos recurrentes.

La presión sobre la tierra es otro de los factores de producción de los deslizamientos en Caracas. A mediados de los años 1970, en pleno boom petrolero de la Venezuela Saudita, los terrenos más asequibles del fondo del valle se encuentran saturados. No quedan más soluciones que ocupar masivamente las colinas del valle de Caracas. Por ley, el material residual de los trabajos de excavación para contar con terrenos planos en la cima de las colinas debe ser evacuado por el constructor. Sin embargo, ahorrando gastos para constructores y ganando terreno vendible para los promotores, en muchos casos el material excavado termina siendo botado al borde de los terrenos, ampliando las superficies planas de construcción y aumentando los rellenos de fuerte pendiente y de material poco consolidado. Esa es precisamente la genealogía del deslizamiento que amenaza la urbanización formal y de clase media de Santa Inés (Figura 5). Para estabilizar el deslizamiento que llega abajo en los patios de las casas, hasta se ha intentado clavar el relleno con pilares de hormigón armado. Pero los pilares no pueden pasar de 30 metros, y no son suficientemente largos como para alcanzar las capas estables debajo del relleno.

\section{Figura 5: Urbanización Santa Inés al pie de un relleno con deslizamiento activo}

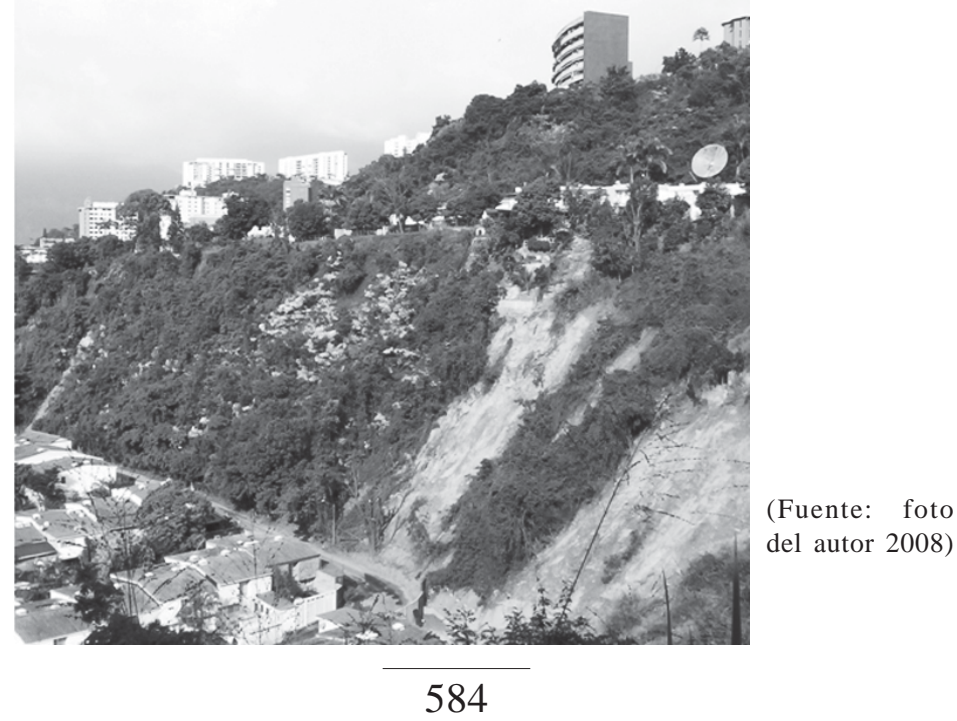


Finalmente, en términos de gestión de riesgo y protección civil, resulta que la manera como se concibe el riesgo y el ambiente, la idea de la vulnerabilidad con la cual se trabaja, y los sistemas implementados en el seguimiento de las situaciones de riesgo son elementos fundamentales a la hora de coordinar una política local de riesgo, y eventualmente disminuir la vulnerabilidad urbana de los sectores más afectados. Las leyes de descentralización de los años 1989 y 1990 vuelven los municipios responsables de sus políticas urbanas y de riesgo. El Distrito Metropolitano de Caracas consta de cinco municipios profundamente marcados por la polarización política nacional de la era Chávez. Las rivalidades políticas cristalizan en la capital nacional, y más que animosidad, es una enemistad abierta que reina entre los municipios del Este por un lado, bastión de la oposición y el municipio Libertador por otro lado, bastión del oficialismo (Rebotier 2011b). En teoría, Caracas cuenta con cinco sistemas diferentes de gestión y seguimiento de riesgo. En teoría, ya que el municipio Libertador resulta mucho menos dotado que los demás municipios, debido a condiciones estructurales de mayor precariedad y escasez de recursos locales. Además de las infraestructuras municipales desiguales, es de notar los diferentes sistemas utilizados en municipios relativamente bien dotados, como lo son los de Chaco y de Baruta (Figura 6), obstaculizando la coordinación de esfuerzos. Las situaciones de riesgo son transversales a las fronteras de los municipios. En algunos municipios, los recursos de gestión de riesgo parecen más favorables que en otros, pero por razones de enemistad política y de fragmentación institucional, la vulnerabilidad de la aglomeración de la capital de Venezuela resulta aumentada.

Figure 6: Fragmentación de los sistemas de identificación y gestión de riesgo

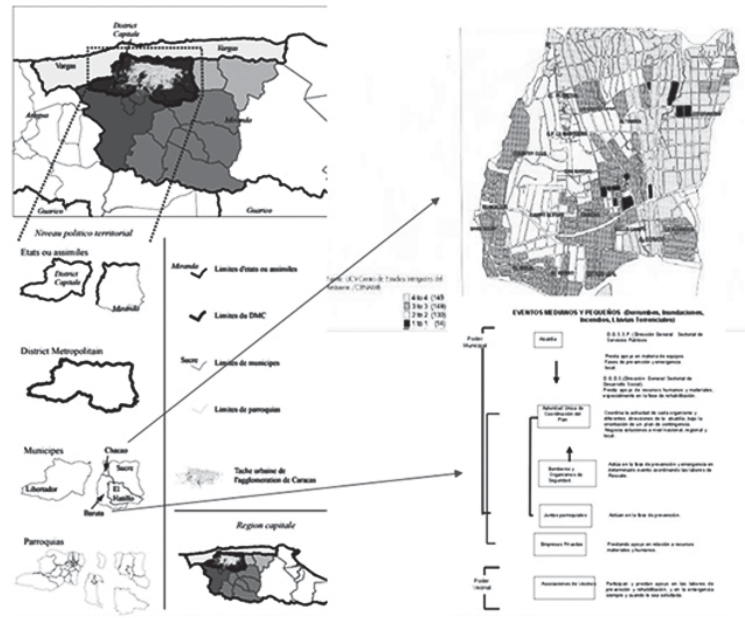

(Fuente: Adaptado de Rebotier 2008) 
En ninguno de estos casos se ha movilizado la pobreza como factor de riesgo. La vulnerabilidad no se reduce a la pobreza, aunque es cierto que la pobreza contribuye en hacer la gente vulnerable mediante muchos otros caminos que la sola distribución desigual de la riqueza. Más que la pobreza, la condición de marginalidad contribuye a la vulnerabilidad (Wisner et al 2004), tanto en la producción de riesgo como en las capacidades de respuesta y en los recursos que se ofrecen para vivir con el riesgo. Ello obliga a situar los estudios de riesgo en un contexto social, histórico y geográfico. También lleva a considerar de manera relativa los factores múltiples que intervienen en la construcción social de las situaciones de riesgo.

\section{Menos vulnerabilidad ¿Para qué? ¿Para quiénes?}

Mientras es notorio como las preocupaciones actuales focalizan más en aspectos técnicos, funcionales y pragmáticos, resulta importante no perder de vista la meta de las iniciativas de gestión de riesgo. Más allá de preguntas tales que ¿Cómo hacer funcionar...? ¿Cómo reducir la vulnerabilidad? ¿Cómo mantener un sistema en función? ¿Cómo volver tal proyecto o política aceptable?, no hay que olvidarse de preguntar ¿Para qué?. De hecho, de concentrarse en la solución técnica a un problema concreto e imperioso, ya no se pregunta sobre ¿qué hace la gente vulnerable? (Cardona 2012). Por ejemplo, para autores entre los más reconocidos de la comunidad de la Resilience Alliance, la resiliencia "no es un ideal” (Gunderson y Holling 2002 : 31). Aunque la noción de resiliencia es objeto de un éxito en parte de la academia y en algunas esferas institucionales internacionales (Rebotier et al 2013), la literatura se concentra en aspectos funcionales y técnicos en vez de investigar sus dimensiones sociales y humanas, sumamente significativas (Janssen y Ostrom 2006). La resiliencia permite mantener el funcionamiento y la estructura de un sistema, y evitar catástrofes, pero no plantea ningún rumbo. No se sustituye a ningún proyecto social, y no justifica evacuar el trabajo eminentemente político que tal proyecto involucra.

Si bien la reducción de los riesgos puede nortear los estudios de riesgo, es importante no prorrogar situaciones de riesgo y fuertes tensiones gracias a unos dispositivos de gestión mejorados. Las conclusiones de un trabajo sobre la gestión de crisis en caso de sismo en Mérida (Venezuela) permiten por ejemplo identificar los mejores terrenos en la ciudad para almacenar material y maximizar la atención a los heridos (Rebotier 2006). Al combinar un modelo de microzonificación sísmica, una evaluación de la vulnerabilidad estructural de los edificio de la ciudad de Mérida, y una estimación de las densidades urbanas, se llega a una distribución potencial aproximada de los sectores urbanos más afectados y a una estimación de los heridos por sismo. El modelo de simulación de crisis sísmica es una herramienta valiosa a la hora de anticipar la gestión de desastre, para la protección civil. El sitio de la ciudad de Mérida se caracteriza por grandes zanjadas y por los tres grandes viaductos que permiten conectar las vertientes de la sierra de la Culata a la terraza aluvial y al corazón de la ciudad pasando por encima del río Albarregas. En la figura 7, se nota el talud que desciende hacia el Chama 
en el primer plano, la terraza aluvial y la pista del aeropuerto, una zanjada verde que corresponde al cañón del Albarregas, y el resto de la aglomeración al pie de la sierra de la Culata en el fondo. En caso de sismo, una de las particularidades de la ciudad de Mérida radica en su incomunicación potencial con el exterior del valle, por un difícil acceso aéreo, y en la posible fragmentación del tejido urbano, en particular entre la terraza y el resto de la aglomeración. Es de suma importancia anticipar las zonas más afectadas, y potencialmente incomunicadas para almacenar material y recursos en previsión de un desastre.

\section{Figura 7: Vista de Mérida hacia el oeste, desde el teleférico}

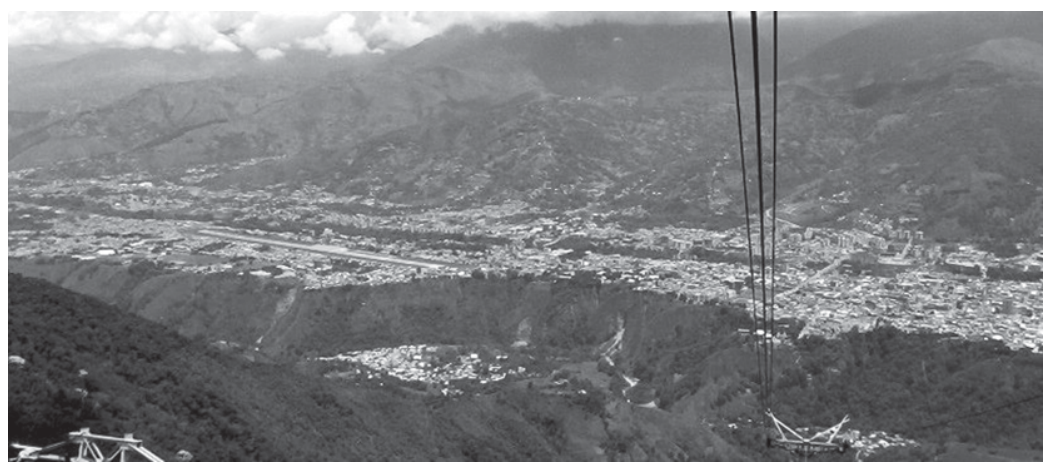

(Fuente: foto del autor 2004)

Sin embargo, aunque de gran importancia, esta herramienta de gestión de crisis no puede contrarrestar el resto de un diagnóstico preocupante en términos de capacidad de respuesta. El anticipar las zonas más afectadas y los focos de heridos, que puede parecer un alivio a la hora de administrar una crisis, bien podría sembrar algunas de las condiciones ulteriores de un riesgo urbano aumentado (Pigeon 2012) al disimular la diversidad de los factores de riesgo. Entre aquellos factores, se puede destacar el control muy débil de la urbanización, la precarización estructural de las viviendas, o el hecho de focalizar la política local de riesgo en la administración de desastres y no en el riesgo como dimensión del desarrollo territorial urbano en el valle andino.

De hecho, el Instituto de Protección Civil y Administración de Desastre del Estado de Mérida (INPRADEM) está marcado por un abordaje "militar" de los riesgos ambientales, como si el desastre fuera una situación de guerra que hubiera que preparar, enfrentar, y superar. Este legado es común a gran parte del continente latino-americano a lo largo del siglo 20 (Revet 2007), hasta las innovaciones institucionales y legales en las cuales Colombia resulta pionera. El INPRADEM es proactivo en la preparación de 
la población urbana, mediante la organización de centros secundarios de rescate, de brigadas o la formación por cursos y talleres. Sin embargo las iniciativas institucionales están dirigidas en mayoría al desastre para minorar los impactos en la ciudad. En esta perspectiva, reducir la vulnerabilidad equivale a reducir los daños potenciales a partir del desastre que se anticipa. Bien se puede hacer grandes inversiones en el “¿como?” hacer un sistema más resistente o flexible en previsión de una crisis para minorar los daños. Si el “¿porqué?” no es también objeto de la política de riesgo, se pierde gran parte de lo que la problemática de riesgo involucra así como de los mecanismos sociales y políticos que se hallan agua arriba de su construcción. La experiencia poco concluyente de la planificación urbana y la provisionalidad relativa de las políticas locales contribuyen a reducir el enfoque sobre riesgo a los aspectos más materiales y de geociencia, dando la ilusión de un control potencialmente mayor y de una neutralidad axiológica de las ciencias al considerar leyes naturales. No hay nada menos cierto cuando partir de la amenaza y focalizar en lo operacional disimula la pluralidad de los factores que explican la construcción de riesgo. De hecho, evita plantear el proyecto comunitario o colectivo como garantía de una reducción sostenible de la vulnerabilidad en el sentido de un desarrollo social más justo e incluyente. Perder confianza en la capacidad social de delinear los contornos de futuros posibles no debe reducir el perímetro de intervención de las ciencias sociales a las maneras como actuar en el presente (Innerarity 2009). Pensar la reducción de la vulnerabilidad en términos de emancipación social permite no reducir los estudios de riesgo a una forma de gestión técnica y a-política, sino ampliar su alcance hacia objetivos de desarrollo (Gaillard y Mercer, 2013) y de transformación social (Pelling 2011).

\section{Conclusión: una mirada desde la vulnerabilidad hacia la emancipación social}

Pensar la reducción de la vulnerabilidad en términos de un proceso emancipador parece relevante (en vista a la literatura crítica sobre riesgos y a los casos de estudio presentados) y prometedor (en vista a los desafíos ambientales contemporáneos que se presentan, en particular en relación con la problemática hegemónica del cambio climático y de las iniciativas y políticas que suscita). Al cuestionar tanto la noción de vulnerabilidad como el trabajo científico de análisis de riesgo, se busca 1- hacer explícita las causas múltiples que hacen - de manera diferenciada - la gente vulnerable, y 2- proponer un abordaje conceptual que permita articular un cambio posible de las relaciones entre sociedades y medio ambiente con un cambio social, en vista a la reducción de riesgos. Es una manera de reanudar con el doble mandato de la crítica de donde emerge la noción de vulnerabilidad: 1una dimensión explicativa, en busca de causas múltiples y necesariamente situadas, y 2- una dimensión transformadora, en busca de un orden socioambiental en redefinición perpetua, menos discriminatorio y menos dañino. Para eso, es necesario reconocer la construcción del riesgo como parte de la organización y del funcionamiento de una sociedad, así como situar el pro- 
pio estudio de riesgo como una actividad social cualquiera, con condicionantes e implicaciones (Rebotier 2012b).

El doble mandato de la crítica puede parecer anacrónico hoy día, como la marca de una época acabada de grandes ficciones y horizontes ideales (Benichou 1977). En tiempo de fracaso de las grandes narrativas, la posmodernidad defiende la duda radical frente a la cual se consolida la necesidad de actuar según criterios técnicos, instrumentales, supuestamente a-políticos, necesarios para dar respuesta a problemas imperiosos (Ellul 1988). De forma más prosaica, en tiempo de crisis, ya no se cuentan los gobiernos de "tecnócratas", más allá de lo político, supuestamente más aptos para solucionar situaciones económicas nacionales inextricables. Abogamos con fuerza para defender ideales normativos -no absolutos, sino constantemente construidos- de emancipación y desarrollo que asume buena parte de la comunidad de académicos en reducción de riesgo (Mercer 2010; Pelling 2011; O’Brien 2012). En apoyo a un abordaje progresista de la vulnerabilidad, es de destacar un rol particular de las ciencias sociales por un lado, y reconocer una necesaria reflexividad y responsabilidad científicas y sociales por otro lado. No se considera la reducción de la vulnerabilidad como una cuestión técnica que hay que implementar, sino como una cuestión social y política de control y acceso al ambiente (o de producción y distribución de los riesgos), objeto democrático de mediación y emancipación social.

- En la perspectiva de un enfoque de ciencias sociales, la caracterización y el análisis de las situaciones de riesgo sólo tienen sentido en relación con las lógicas sociales a las cuales se pueden vincular. El trabajo de situación y de politización tanto de los factores de riesgo como del abordaje analítico que se moviliza es esencial ya que son condicionantes de los riesgos, i.e. los valores imperantes que sirven par discriminar y evaluar, las convenciones, lo que es importante y para quiénes, son elementos claves. Aquellos elementos orientan la manera como se formatean los problemas ambientales y sus potenciales soluciones en el contexto de un debate tenso sobre el ambiente, a diferentes escalas, que tiende a naturalizar y tecnificar los desafíos pendientes (Toledo 2012).

- Pero, además de una necesaria autonomía conceptual de las ciencias sociales críticas a la hora de pensar el ambiente en sociedad, está un reto de reflexividad y responsabilidad (Jonas 1995 [1979]). Aquel reto se plantea en las ciencias y en el trabajo de investigación (para salir de un nacionalismo metodológico en el ámbito de una problemática ambiental global, Beck 2007). También se plantea entre las sociedades, en términos de relaciones al medio ambiente, de organización y dinámicas sociales. De hecho, en términos de acción colectiva, y mientras que los grandes actores modernos legítimos como el Estado, la ciencia, o las instituciones establecidas son fuertemente cuestionados (Theys 2003), el papel político que los individuos pueden desempeñar parece de suma importancia en un período de recomposición de la subjetividad y de una articulación cuestionada al colectivo (della Porta y Piazza 2008; Leff 2010; Mac Adam y Boudet 2012). 
Polis, Revista Latinoamericana, Volumen 13, $N^{\circ}$ 38, 2014

\section{Notas}

${ }^{1}$ Una versión oral corta de este artículo fue presentada en el $7^{\circ}$ CEISAL - Porto (Portugal), 12-15 de junio 2013.

${ }^{2}$ Se usa la palabra progresista para resaltar la dimensión transformadora de un abordaje crítico. De hecho, para muchos, y siguiendo un uso debilitado del adjetivo, crítico hace referencia a una posición argumentativa que cuestiona un determinado postulado, sin necesariamente elaborar un postulado contradictorio. Aquella concepción de una crítica "en reacción" no deja espacio para las ambiciones transformadoras que caracterizan los estudios críticos de riesgo a mediados de la segunda mitad del siglo 20 en las Américas, Norte y Sur (Pelling 2011; O’Brien 2012). El uso del adjetivo progresista es una manera de reanudar con una concepción doble de la crítica en el mundo francófono (cuando se usa el adjetivo radical en el mundo anglófono): la crítica cuestiona el mundo tanto como ofrece cambiarlo (De Koninck 1984).

${ }^{3}$ http://www.desenredando.org/ (23 de marzo 2013).

${ }^{4}$ Acta del presidente José Antonio Páez. Interior y Justicia. Tomo LIII, 1832. Folio 247

${ }^{5}$ 27/10/1880. Secretaria de Interior y Justicia. Tomo MXXV. Folio 32.

${ }^{6}$ Información concedida por un responsable de la protecciòn civil metropolitana de Caracas, 2005. 


\section{Bibliografía}

Beck, U. (2003 [1986]), La société du risque. Sur la voie d'une autre modernité. Flammarion, Paris.

Ídem (2007), "La condition cosmopolite et le piège du nationalisme méthodologique”, en Wieviorka, M. (ed.), Les Sciences sociales en mutation. Editions Sciences Humaines, Paris, 223-237.

Benichou, P. (1977), Le temps des prophètes, doctrines de l'âge romantique. Gallimard, Paris.

Bonnal, Ph.; Bonin, M. y Aznar, O. (2012), “Les évolutions inversées de la multifonctionnalité de l'agriculture et des services environnementaux”, en VertigO - la revue électronique en sciences de l'environnement, vol 12, n³, http://vertigo.revues.org/12882 (14 de abril 2013).

Buitrago Segura, L. (1980), Caracas la horrible. El ateneo de Caracas, Caracas.

Burton, I. y Kates, R. (1964), “The Perception of Natural Hazards in Resource Management”, en Natural Resources Journal, vol 3, n³, 412441.

Cardona, O.D. (2012), “Determinants of risk: exposure and vulnerability”, en Managing the risks of extreme events and disasters to advance climate change adaptation. A special report of working groups I and II of IPCC, Cambridge University Press - Editions de la MSH, Cambridge - New York, 65-108.

Castillo, O. (1990), Los años del bulldozer. Ideología y política, 1958-1958. Editorial Tropykos. Caracas.

Coronil, F. (1997), The magical state. Nature, money and modernity in Venezuela, The University of Chicago Press, Chicago and London.

de Armas Chitty, J.A. (1967), Caracas, origen y trayectoria de una ciudad. Fundación Creole, Caracas.

De Koninck, R. (1984), “La géographie critique”, en Bailly, A. (dir.) Les concepts de la géographie humaine. Masson, Paris, 121-131.

della Porta, D. y Piazza, G. (2008), Voices of the Valley, Voices of the Straits: How Protest Creates Community. Berghahn Books, Oxford.

Ekers, M. y Loftus, A. (2013), "Revitalizing the production of nature thesis: A Gramscian turn?”, en Progress in Human Geography, vol. 37, $\mathrm{n}^{\circ} 2,234-252$. 
Ellul, J. (1988), Le bluff technologique. Hachette, Paris.

Fogelson, R. (2005), Bourgeois Nightmares: Suburbia 1870-1930. Yale University Press, New Haven.

Forsyth, T. (2012), "Politicizing environmental science does not mean denying climate science nor endorsing it without question”, en Global Environmental Politics, vol 12, n², 18-23.

Gaillard, J.-C. y Mercer, J. (2013), "From knowledge to action: bridging gaps in disaster risk reduction”, en Progress in Human Geography, vol 37, $\mathrm{n}^{\circ} 1,93-114$.

Gallegos, R. (1929), Doña Barbara. Editorial Araluce, Barcelona.

García Acosta, V. (2005), “El riesgo como construcción social y la construcción social de riesgos”, en Desacatos, vol 19, 11-24.

Giddens, A. (1994 [1991]), Les conséquences de la modernité. L'Harmattan, Paris.

Godelier, M. (1984), L’idéel et le matériel. Fayard, Paris.

González Casas, L. (2002), "Caracas: Territory, architecture and urban space”, en Almandoz, A. (ed.), Planning Latin America's capital cities, 1850-1950. Routledge, London, 114-140.

Gunderson, L. y Holling, C.S. (2002), Panarchy. Understanding transformations in human and natural systems. Island Press, Washington.

Hewitt, K. y Burton, I. (1971), The hazardousness of a place: a regional ecology of damaging events. University of Toronto Press, Toronto.

Innerarity, D. (2009), El futuro y sus enemigos. Una defensa de la esperanza política. Editorial Paidós, Barcelona.

Janssen, M.A. y Ostrom, E. (2006), "Resilience, vulnerability, and adaptation: A cross-cutting theme of the International Human Dimensions Programme on Global Environmental Change”, en Global Environmental Change, vol 16, n³, 237-239.

Jonas, H. (1995 [1979]), El principio responsabilidad. Ensayo de una ética para la civilización tecnológica. Herder, Barcelona.

Latour, B. (1994), Nous n'avons jamais été modernes. Essai d'anthropologie symétrique. La Découverte, Paris.

Lefebvre, H. (2000 [1974]), La production de l'espace. Anthropos, Paris. 
Leff, E. (2010), "El desvanecimiento del sujeto y la reinvenciòn de las identidades colectivas en la era de la complejidad ambiental”, en Polis. Revista Latinoamericana, vol 27, http://polis.revues.org/862 (23 de marzo 2013).

Mac Adam, D. y Boudet H. (2012), Putting Social Movements in their Place: Explaining Opposition to Energy Projects in the United States, 20002005. Cambridge University Press, Cambridge.

Mercer, J. (2010), “Disaster risk reduction or climate change adaptation: are we reinventing the wheel?”, en Journal of International Development, vol 22, 247-264.

Musset, A. (2002), Villes nomades du nouveau monde. Editions de l'EHESS, Paris.

O’Brien, K. (2012), “Global environmental change II: from adaptation to deliberate transformation”, en Progress in Human Geography, vol 36, n5, 667-676.

O’Brien, K.; Eriksen, S.; Nygaard, L. y Schjolden, A. (2007), “Why different interpretations of vulnerability matter in climate change discourses", en Climate Policy, vol 7, n²1, 73-88.

Pelling, M. (2011), Adaptation to climate change.From resilience to transformation. Routledge, London and New York.

Pigeon, P. (2012), Paradoxes de l'urbanisation. Pourquoi les catastrophes n'empêchent-elles pas l'urbanisation? L'Harmattan, Paris.

Rebotier, J. (2006), “Les risques sismiques à Mérida (Venezuela): pour une association des communautés à la prévention des catastrophes dans les sociétés fragiles”, en Cahiers des Amériques Latines, vol 47, n³, 109-122.

Ídem (2008), Les territorialités du risque urbain à Caracas. Les implications d'un construit socio-spatial dans une métropole d'Amérique latine. Thèse de doctorat en géographie, IHEAL-Paris 3.

Ídem (2011a), “Habiter les territoires et construire les risques, entre empreintes spatiales et logiques sociales - Caracas”, en November, V.; Penelas, M. y Viot, P. (eds.) Habiter les territoires à risque. Presses Polytechniques et Universitaires Romandes, Lausanne, 81-100.

Ídem (2011b), "Politicizing fear of crime and insecurity in Caracas. The manufacturing of a fearful urban meta-narrative”, en Emotion, Space and Society, vol 4, n², $104-112$.

Ídem (2012a), “La production de l'environnement”, comunicación en el coloquio internacional Ecologie, Géographie, Politique: un climat de 
changement, Orléans (Francia), 6-9 de septiembre.

Ídem (2012b), "Vulnerability conditions and risk representations in LatinAmerica: framing the territorializing urban risk”, en Global Environmental Change, vol 22, n², 391-398.

Rebotier, J.; López-Peláez, J. y Pigeon, P. (2013), “Las paradojas de la resiliencia: miradas cruzadas entre Colombia y Francia”, en Territorios, vol 28, 127-145.

Revet, S. (2011), Injonctions contradictoires. La gestion internationale des catastrophes “naturelles”, entre vulnérabilité et résilience. Les études du CERI, Paris.

Ídem (2007), Anthropologie d'une catastrophe. Les coulées de boue de 1999 au Venezuela. Presses de la Sorbonne Nouvelle, Paris.

Ribot, J. (2009), "Vulnerability does not fall from the sky: toward multiscale, pro-poor climate policy”, en Mearns, R. y Norton, A. Social dimension of climate change. Equity and vulnerability in a warming world. World Bank, Washington, 47-74.

Serres, M. (1990), Le contrat naturel. François Bourin, Paris.

Simonet, G. (2009), “Le concept d'adaptation: polysémie interdisciplinaire et implication pour les changements climatiques", en Natures Sciences Sociétés, vol 17, n4, 392-401.

Singer, A. (1983), “Geomorfología, arqueología y aspectos de geología reciente”, en Boletín Informativo Asociación Venezolana de Arqueología, vol 2, $n^{\circ} 2,6-44$.

Smith, N. y O’Keefe, P. (1980), “Geography, Marx and the concept of nature”, en Antipode, vol 12, n², 30-39.

Theys J. (2003), “La gouvernance entre innovation et impuissance. Le cas de l'environnement”, en Développement durable et territoires, Dossier 2: http://developpementdurable.revues.org/1523 (23 de marzo 2013).

Toledo, V. (2012), “Diez tesis sobre la crisis de la modernidad”, en Polis. Revista Latinoamericana, vol 33, http://polis.revues.org/8544 (23 de marzo 2013).

White, G. (1958), Changes in urban occupancy of flood plains in the United States. University of Chicago, Chicago.

Wisner, B. (1976), Man-made famine in Eastern Kenya: the interrelationship of environment and development. Institute of development studies at the University of Sussex, Brighton, England. 
Wisner, B.; Blaikie, P.; Cannon, T. y Davis, I. (eds.), (2004 [1994]), At risk, natural hazards, people's vulnerability and disasters. Routledge, London.

Young, O.; Berkhout, F.; Gallopin, G.; Janssen, M.; Ostrom, E. y van der Leeuw, S. (2006), “The globalization of socio-ecological systems: an agenda for scientific research”, en Global Environmental Change, vol 16, n³, 304-316.

Recibido: 11.04.2013

Aceptado: 15.11.2013 